\title{
A mathematical model of particle retention in the air-spaces of human lungs
}

\author{
T R GERRITY, C S GARRARD, AND D B YEATES
}

From the Section of Environmental Medicine, Department of Medicine, University of Illinois, Abraham Lincoln School of Medicine, and the West Side Veterans Administration Hospital, Chicago, Illinois 60612, USA

\begin{abstract}
Knowledge of the total and regional lung retention of particles inhaled continuously by man over long periods can be useful in understanding the potential role of inhaled particles in the pathogenesis of lung diseases. Owing to practical and ethical considerations, however, little or no experimental information exists. A mathematical model of particle retention simulating environmental and occupational exposures has therefore been developed that takes into account particle deposition, tracheobronchial clearance, and two phases of alveolar clearance in the Weibel A anatomical lung model. The derived equations of retention kinetics predict retention of particles as a function of exposure time. For a continuous exposure (simulating environmental conditions) to $4 \mu \mathrm{m}$ particles, the model predicts that retained particles approach an equilibrium between deposited and cleared particles with the $95 \%$ level being reached in 293 days. For an intermittent exposure (simulating occupational conditions) equilibrium is approached in five years. The whole lung burden of particles is predicted to be $9 \%$ of the total mass that entered the lung after a oneyear environmental exposure and $1.5 \%$ after a 25 -year occupational exposure. The equilibrium surface concentration and integrated dose of particles per airway generation predict enhanced risk to the pathogenic effects of inhaled particles in the large airways and respiratory bronchioles.
\end{abstract}

Continuous exposures of the human lung to toxic particles from environmental and occupational sources have been implicated in the pathogenesis of many lung diseases. The focal nature of some of these diseases may be related to regional variations of particle exposure in the lung.

Experimental human studies of the long-term retention of particles inhaled continuously are difficult to conduct because of practical as well as safety considerations for volunteers. Cohen, ${ }^{1}$ Cohen et al, ${ }^{2}$ and Kalliomäki et $a{ }^{\beta}$ have measured the long-term retention of magnetite in the whole lung of occupational workers by magnetic techniques. The dynamics of regional accumulation of particles, however, cannot be measured.

An alternative to human investigation has been the use of mathematical models of deposition and clearance, ${ }^{4-13}$ but these predict only the distribution of particles immediately after a single breath. Brain and

This project was supported by Oak Ridge National Laboratory Basic Agreement No 7762 and National Institutes of Health Grant No 13824 awarded by the National Heart, Lung and Blood Institute, and the Medical Research Service of the Veterans Administration.

Received 4 January 1982

Accepted 24 March 1982
Valberg ${ }^{14}$ developed a mathematical model of retention of continuously inhaled particles using the ICRP task force .. odel of the lung. ${ }^{15}$ In this model the lung was divided into four regions with the ciliated airways and alveoli each comprising one large compartment.

In this paper we present a mathematical model of the regional retention of continuously inhaled particles in the 23 generations of the Weibel A model of the human lung ${ }^{16}$ and integrate into it available experimental data. It takes into account the simultaneous processes of deposition and clearance in each airway generation. Deposition fractions are calculated using the mechanisms of inertial impaction, gravitational sedimentation, and Brownian diffusion. Tracheobronchial clearance from each airway generation is modelled after a technique developed by Lee $e t$ al. ${ }^{17}$ Alveolar clearance is represented as a two-component exponential with short-term and long-term components. The processes of deposition and clearance are joined together by viewing deposition as a particle "source" in a set of differential equations governing particle clearance. ${ }^{17}$

Results for the accumulation of particles as a function of time of exposure, regional surface concentration of particles, and integrated dose are 
shown, as well as the quantitative differences between continuous and intermittent exposures. The effects of particle size, breathing pattern, and variations in lung morphology are also considered.

\section{Model formulation}

The determination of the number of particles present in any airway generation, $\alpha$, at any time, $t$, after the onset of exposure, is conceptually straightforward. The net rate of change of particle number in airway generation $\alpha$ (designated $R_{\alpha}^{\text {net }}$ ) is the difference between the rate at which particles enter the generation and the rate at which they leave. This can be represented symbolically as

$$
\mathbf{R}_{\alpha}^{\text {net }}=\mathbf{R}_{\alpha}^{\text {in }}-\mathbf{R}_{\alpha}^{\text {out }}
$$

$\mathbf{R}_{\alpha}^{\text {in }}$ depends on the distribution of particles distal to $\alpha$, the transport rates of particles in generations distal to $\alpha$, and the time after the onset of exposure. It also depends on the rates at which particles are deposited in generation $\alpha$ from the external environment. $R_{\alpha}^{\text {out }}$ depends on the transport rate of particles within $\alpha$ and the time after onset of exposure.

The number of particles in any airway generation $\left(\mathrm{N}_{\alpha}(\mathrm{t})\right)$ at any time after onset of exposure is obtained by integration of $R_{\alpha}^{\text {net }}$ in analogy with the relationship between airflow and volume. The total number of particles present in the lungs $\left(N^{\text {tot }}(t)\right)$ is the sum of all $N_{\alpha}(t)$.

The retention kinetics of continuously inhaled particles may be determined by breaking the formulation into three parts as shown in fig 1. Pathway " $a$ " refers to particles deposited in the tracheobronchial tree and cleared by mucociliary clearance. Pathway "b" refers to particles deposited in the alveoli and transported to the ciliated airways. Particles deposited in the alveoli and cleared by the slow phase follow pathway "c."

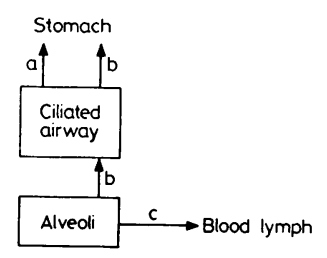

Fig 1 Pathways of particle clearance considered in retention model. Pathway " $a$ " refers to particles deposited on mucus blanket and removed by mucociliary clearance. Pathway " $b$ " refers to particles deposited in alveoli and transported by fast phase of alveolar clearance to mucociliary escalator. Pathway " $c$ " refers to particles deposited in alveoli and removed by slow phase of alveolar clearance to blood and lymphatic systems.
The formulation of a mathematical model of particle retention in the human lung thus requires a set of basic assumptions concerning lung morphology, rates of particle transport in the airways, and alveolar clearance.

For morphological data the Weibel A model of the lung $^{16}$ is used in which the lung is pictured as a symmetric, dichotomously branching system of airway generations. The generations are labelled from 0 (the trachea) to 23 (the terminal alveolar sacs) with the ciliated airways extending to generation 15 (the terminal bronchioles).

Particle transport rates in each generation of the ciliated airways are calculated by assuming a uniformly deep mucus blanket with no net secretion or absorption of mucus within an airway generation with the exception of the distal airways where the mucus is assumed to be supplied. The transport rate of particles $\left(v_{\alpha}\right)$ in any airway generation $(\alpha)$ is then related to the transport rate in the trachea $\left(v_{0}\right)$ by the relation $v_{\alpha}=v_{0} d_{0} / 2^{\alpha} d_{\alpha}$ where $d_{\alpha}$ is the diameter of an airway in generation $\alpha$. It must be emphasised that this is not a model of mucus transport. The aforementioned equation is only a means of expressing particle transport rates. Mucociliary transport of particles is the only mechanism of tracheobronchial clearance considered here. Clearance of particles through the tracheobronchial epithelium is not taken into account and is assumed to represent a small fraction of the total amount of particulate cleared.

Alveolar clearance is represented by a two-component exponential with a rapid component (possibly due to clearance by macrophages ${ }^{18}$ and free particle transport) and a slow component due to mechanisms such as dissolution. ${ }^{19}{ }^{20}$ In this paper the case of retention of moderately retained particles as defined by the ICRP task force ${ }^{15}$ is considered. The clearance half-time of the rapid phase is set equal to 24 hours, and the slow phase is set equal to 68 days..$^{18}$ In addition, particles cleared by the rapid phase are assumed to be transported from each alveolated generation directly to the mucociliary escalator. Particles cleared by other mechanisms are assumed to be eliminated from the alveoli and transported to other organs of the body. Forty per cent of the particles deposited in the alveoli are assumed to clear by rapid processes and the remaining $60 \%$ by slower processes. ${ }^{15}$ The fractional deposition per airway generation is computed using the calculations of Gerrity et al, ${ }^{21}$ which use the mechanisms of inertial impaction, gravitational sedimentation, and Brownian diffusion. ${ }^{9}$ Deposition is calculated for a complete respiration cycle including a pause between each phase. Discussion of the validity and limitations of these assumptions is reserved until the last section.

Some qualitative observations may be made from 
the structure of the model in equation (eq) 1 . At the onset of exposure the number of particles present in the lungs is zero. After the beginning of exposure, the number of particles in the lungs increases since the rate of particle deposition exceeds the rate of removal by clearance mechanisms. As the exposure continues and the number of particles in the lungs increases, the rate of particle removal naturally increases. Provided that the mechanisms of removal are not affected by the number of particles, the rate of removal will eventually equal the rate of deposition. When this happens the net rate of change of particle number equals zero and the particle number within the lungs reaches a constant. Similarly, the particle number in each airway generation reaches a constant level. Hereafter these constant retention levels will be referred to as "equilibrium" levels and designated as $\mathrm{N}^{\text {tot,eq }}$ and $\mathrm{N}_{\alpha}^{\text {eq }}$.

Several phenomena may be estimated by this approach to retention kinetics. Computation of $\mathrm{N}^{\text {tot.eq }}$ for different particle sizes and atmospheric particulate concentrations can give estimates of total lung burdens of inhaled particles. The time taken to reach $95 \%$ of $\mathrm{N}^{\text {tot.eq }}$ can provide an estimate of the time it takes an individual's lungs to accumulate its maximum lung burden. The surface concentration of retained particles on air-space lumen may be important when considering health effects of inhaled particles. The surface concentrations within each generation are easily computed by dividing particle number by luminal surface area (as computed from the Weibel A dimensions). Of particular interest is the surface concentration at equilibrium designated as $\mathrm{C}_{\alpha}^{\mathrm{eq}}$. The number of particles per unit surface area that passes over the tissue after a time t may also have a bearing on health effects, and this may be computed from the surface concentration by a further integration over time. This quantity is called the "integrated particle dose" and is designated as $D_{\alpha}(t)$. The longer the time after the onset of exposure, the higher the integrated dose will be. By the time particle number approaches equilibrium, the integrated dose per generation is increasing linearly with time.

The mathematical derivations of all the aforementioned quantities are presented in the appendix. The interested reader may want to study these, though it is not necessary for a general understanding of this paper.

\section{Application of the model}

The model is first applied to the continuous inhalation of a $4 \mu \mathrm{m}$ aerodynamic diameter aerosol with a tidal volume of $700 \mathrm{~cm}^{3}$ and a frequency of 25 breaths a minute (minute volume of $17.5 \mathrm{l}$ ). The breathing is assumed to follow a square wave pattern. The rate of transport of particles in the trachea $\left(\mathrm{v}_{0}\right)$ is set equal to $5.5 \mathrm{~mm} / \mathrm{min}$, in agreement with previous models ${ }^{17}$ and experimental studies. ${ }^{22-24}$ The retention half times for the fast and slow components of alveolar clearance are given the values of 24 hours and 68 days respectively. Retention is calculated as a fraction of the particles deposited in a single respiration cycle and is thus expressed as retained mass/inhaled single breath mass.

Figure 2 shows the accumulation of particles as a function of time $\left(\mathrm{N}^{\text {tot }}(\mathrm{t})\right)$ during the first 120 hours of a continuous exposure. Because of the rapid clearance of the ciliated airways, retention in these airways approaches an equilibrium condition whereas retention in the alveolar regions continues to increase almost linearly. At 103 hours, the ciliated airways reach $95 \%$ of their equilibrium retention value of $5.8 \times 10^{3}$.

Figure 3 shows the accumulation of particles in the whole lung model over a period of 240 days. Here it

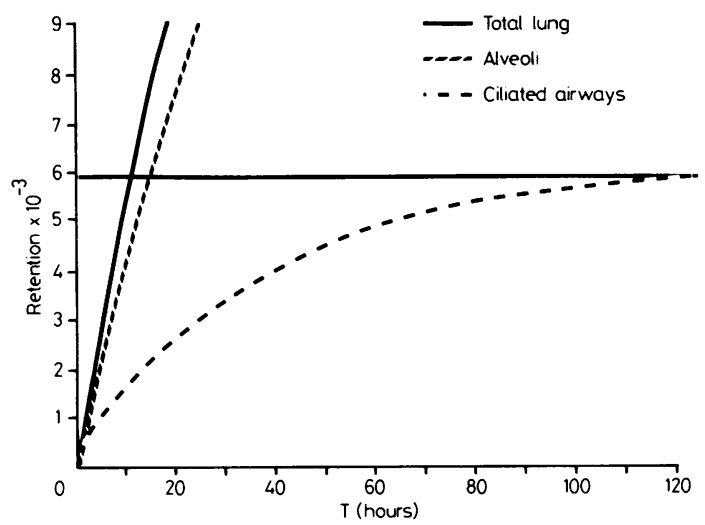

Fig 2 Accumulation of particles in lung during first 120 hours of a continuous exposure. Horizontal line represents equilibrium retention of particles in ciliated airways.

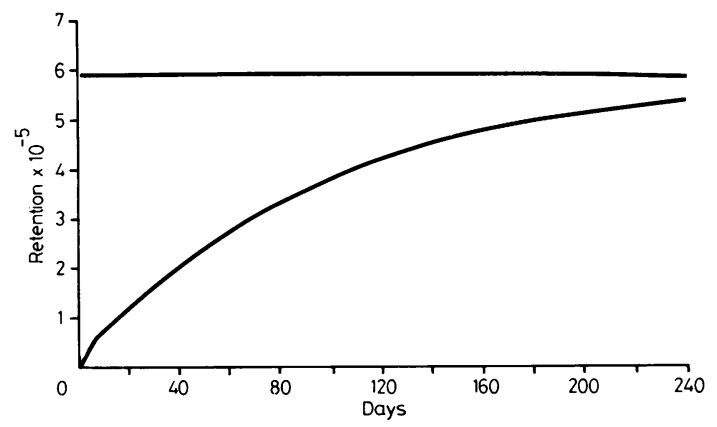

Fig 3 Accumulation of particles in lung during first 240 days of a continuous exposure. Horizontal line represents equilibrium retention of particles in whole lung. 
can be seen that $N^{\text {tot }}(t)$ approaches a condition of equilibrium. The time required for $N^{\text {tot }}(t)$ to reach $95 \%$ of the equilibrium value $\left(\mathrm{N}^{\text {tot,eq }}\right)$ of $5.9 \times 10^{5}$ is 293 days.

The equilibrium retention value of $5.9 \times 10^{5}$ is important in that it gives a predicted measure of retained mass for a long-term continuous exposure. If a $100 \mu \mathrm{g} / \mathrm{m}^{3}$ concentration of particles enters the trachea the equilibrium retention predicted by the model equals $41 \mathrm{mg}$. If no clearance had taken place the total mass of particles retained in the lung after one year would have equalled $454 \mathrm{mg}$ and would be linearly increasing.

So far, only continuous exposure has been considered, but occupational workers are exposed to particles on an intermittent basis. The model is readily adapted to allow for weekends and periods of eight hours of continuous exposure followed by periods of 16 hours of clearance without deposition. In these circumstances retention increases much more slowly than in the continuous case (fig 4), and equilibrium is achieved in about five years.

The equilibrium surface concentration $\left(\mathrm{C}_{\alpha}^{\text {eq }}\right)$ and integrated dose $\left(D_{\alpha}(t)\right)$ per airway generation at 293 days have been calculated using eq 11A. Surface concentration is shown in fig 5 and integrated dose in fig 6. Calculations have been included for two other combinations of particle size and breathing pattern: $10 \mu \mathrm{m}$ particles inhaled with a tidal volume of 1000 $\mathrm{cm}^{3}$ and a frequency of 30 breaths a minute (minute volume of $30 \mathrm{l} / \mathrm{min}$ ); and $0.5 \mu \mathrm{m}$ particles inhaled with a tidal volume of $400 \mathrm{~cm}^{3}$ and a frequency of 15 breaths a minute (minute volume of $6 \mathrm{l} / \mathrm{min}$ ). Breath pauses are not included in any of the cases. These choices produce a wide range of single breath deposition patterns within the lungs. The results are normalised to the particle number in the tidal volume of the $0.5 \mu \mathrm{m}$ case. Both surface concentration and

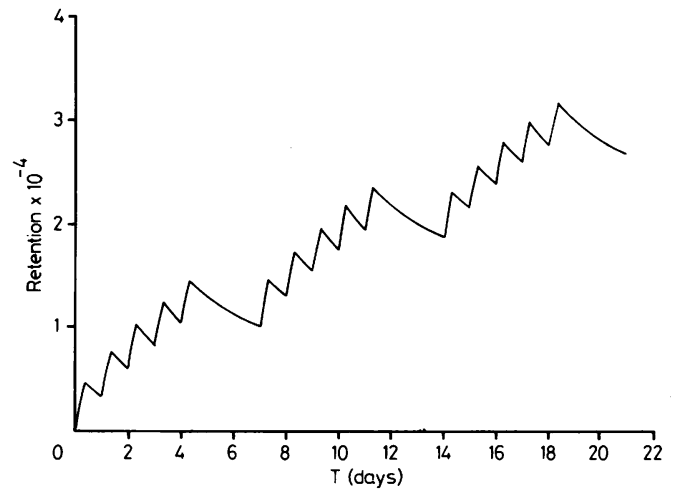

Fig 4 Accumulation of particles in lung during first 22 days of an intermittent (occupational) exposure. integrated dose follow similar patterns with the highest concentrations and doses occurring at generation 16 (the respiratory bronchioles). The surface concentration and integrated dose for the $10 \mu \mathrm{m}$

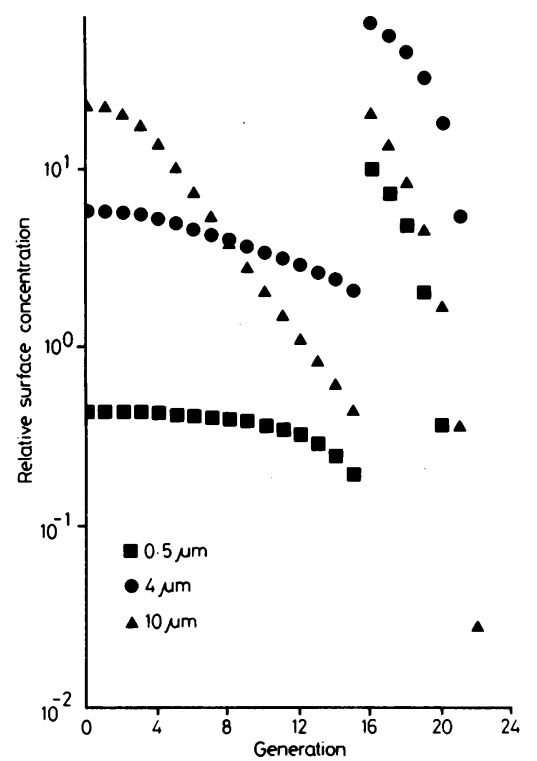

Fig 5 Surface concentrations of particles in each airway generation at equilibrium for a $0.5 \mu \mathrm{m}, 4.0 \mu \mathrm{m}$, and 10.0 $\mathrm{\mu m}$ aerosol. See text for details of breathing pattern.

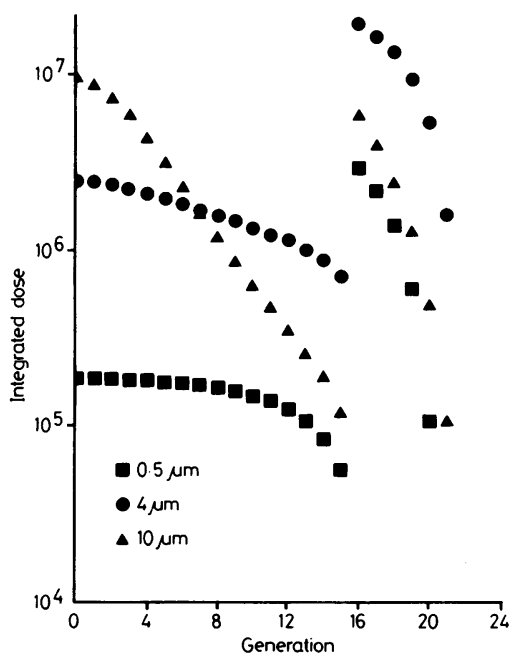

Fig 6 Integrated doses of particles in each airway generation at 293 days for a $0.5 \mu \mathrm{m}, 4.0 \mu \mathrm{m}$, and 10.0 um aerosol. See text for details of breathing pattern. 
particles is highly peaked at the trachea and falls off nearly two orders of magnitude toward the terminal bronchioles. On the other hand, the surface concentration and integrated dose of the $0.5 \mu \mathrm{m}$ particles is a relatively flat distribution throughout the ciliated airways. The distribution of surface concentration and integrated dose for the $4 \mu \mathrm{m}$ particles is intermediate between the others in the large airways but is greatest in the small airways. The apparent discontinuity between retention in the ciliated airways and alveoli is an effect of the differing time scales over which clearance takes place in these two regions as well as the symmetric nature of the Weibel A lung model. This has been noted in other dosimetric models. ${ }^{14}$ The equilibrium surface concentration is illustrated since it represents the endpoint of an exposure, and the dose at 293 days is shown because $95 \%$ of equilibrium is attained at this time for the $4 \mu \mathrm{m}$ exposure. The one important distinction that should be made between these two figures is that, since according to eqs $18 \mathrm{~A}-20 \mathrm{~A}$ the dose increases proportional to the equilibrium concentration, regions of highest equilibrium concentration will receive relatively higher doses as time increases.

Particle size and breathing pattern are extrinsic quantities that can be specified when making estimates of particle uptake. The lengths and diameters of airways in the lungs cannot be defined with such confidence. The Weibel model is used as an approximation to the average human lungs. The effect of deviations of airway geometry away from this average is investigated by allowing the diameters and lengths of airways to vary by an amount $\Delta d$ and $\Delta l$ respectively. Figure 7 shows total lung equilibrium retention as a function of fractional linear dimension changes assuming that $\Delta \mathrm{d} / \mathrm{d}=\Delta \mathrm{l} / \mathrm{l} \equiv \Delta \mathrm{x} / \mathrm{x}$ for the $4 \mu \mathrm{m}$ case. The peak retention occurs when the linear

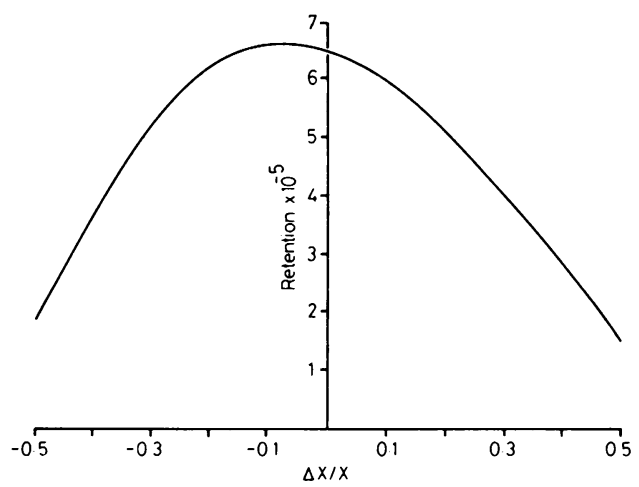

Fig 7 Equilibrium particle retention versus fractional change in linear dimensions of airways. dimensions of the conducting airways are $10 \%$ smaller than those given in the Weibel model. In the case of larger dimensions fewer particles are deposited in each breath, whereas in the case of smaller dimensions more and more of the particles are deposited on the ciliated airways from which they can clear faster than from alveoli. Figure 8 shows the range of surface concentrations at equilibrium predicted for variations of $\pm 30 \%$ of conducting airway dimensions away from the Weibel dimensions. Although the smaller dimensions dictate higher deposition in large airways and lower equilibrium retention, the smaller surface area on which the particles deposit results in high surface concentrations in the conducting airways.

\section{Discussion and conclusions}

Every day the human lung is exposed to a wide variety of particulate insults as a consequence of environmental air pollution. The resulting pathological response of the lung can range over a broad range of disease from pneumoconioses to chronic airways obstruction. These responses are related to the physicochemical nature of the particles and the distribution of particles in the lung.

In this paper a new model of long-term accumulation and retention of continuously inhaled particles has been proposed, which gives detailed information on the relative surface concentration and time integrated dose in each of the 23 generations of the Weibel model. ${ }^{19}$

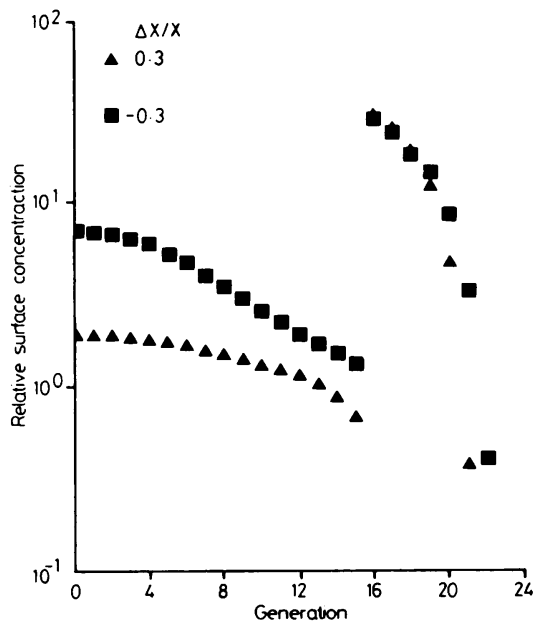

Fig 8 Equilibrium surface concentrations of inhaled particles for $\pm 30 \%$ changes in linear dimensions of airways. 
The derivation and application of the model has required the use of some assumptions. Errors in the morphology, deposition, and clearance assumptions are likely to induce quantitative differences in the results, though many qualitative aspects should remain the same.

The choice of the Weibel lung model facilitates the deposition calculations. A previous publication of this group ${ }^{21}$ showed that reasonable agreement between the deposition calculations and experimental values for total lung deposition could be obtained with the Weibel model. Recent data on alveolar deposition of radiolabelled ferric oxide particles ${ }^{25}$ show the regional validity of the calculations (fig 9). The primary disadvantage of the Weibel model is its inherent anatomical inaccuracy due to its symmetry. Variations of $\pm 30 \%$ in linear airway dimensions are seen in fig 8 to produce two to threefold changes in equilibrium surface concentration. This variability is less, though, than the variability potentially induced by different particle sizes and breathing patterns.

The equations governing retention in the ciliated airways are approximate in nature. They represent the kinetics of retention as an average over each airway generation. Lobar variations in tracheobronchial clearance cannot be predicted. The use of transport rates that vary inversely with airway generation circumference has been tested with some success. ${ }^{17}{ }^{23}$ The model predicts a $4.6 \mu \mathrm{m} / \mathrm{min}$ transport rate in the terminal bronchioles if the tracheal mucus transport rate is $5.5 \mathrm{~mm} / \mathrm{min}$. It is known from in vitro studies ${ }^{26}$ that mucus transport rates in distal generations are slower than the rate in the trachea. The predicted decrease of transport rates in proportion to surface area is only a means to represent the transport rates, which in turn give reasonable agreement between predicted and experimentally

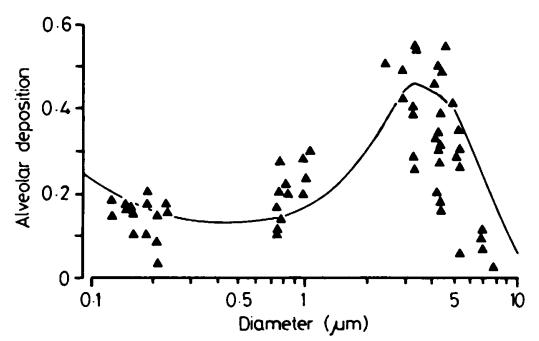

Fig 9 Model prediction of fraction of deposited particles in alveoli (solid line) compared with alveolar deposition data (triangles) as a function of particle size gathered by Lippmann et al. ${ }^{25}$ Horizontal axis is mass median aerodynamic diameter above $0.5 \mu \mathrm{m}$ and mass median diameter below $0.5 \mu \mathrm{m}$. Aerosols were inhaled with an average inspiratory flow rate of $467 \mathrm{~cm}^{3} / \mathrm{s}$ and tidal volume of $1000 \mathrm{~cm}^{3}$. measured clearance curves. ${ }^{17}$ If the decrease in rates is not this dramatic, figs 5 and 6 would show a relative increase in concentration and dose in the large airways.

The predictions of alveolar retention are based on measurements of alveolar clearance. Most studies of alveolar clearance in man have found a long-term phase that lasts anywhere from a few days to over 1000 days. ${ }^{15}{ }^{18-20}$ This range may be due to differences in the physicochemical properties of the particles. The long-term retention half time of inhaled iron oxide particles has been found to equal $68( \pm 5)$ days. ${ }^{27-29}$ This retention half time was chosen as a suitable intermediate value to illustrate the model. The short, 24-hour half time alveolar clearance phase was chosen since this phase has been observed in association with measurements of longer clearance times. Evidence exists ${ }^{18}$ that this rapid clearance may be due to macrophage engulfment and transport toward the mucus blanket. Consistent with these views, $60 \%$ of alveolar deposited particles were assumed to clear by slow processes and $40 \%$ by a rapid phase. The particles that clear by the slow processes are assumed to be transported directly into the blood and lymph. Since little is known of the detailed kinetics of particle transport in the alveoli, the rapid phase of clearance is viewed as a two-step process in which particles are first transported directly from the alveoli to the terminal bronchioles, and then are removed by mucociliary clearance toward the trachea. The question of transport from one alveolus to another is avoided. This simplification probably causes a small underestimation of dose and concentration in the respiratory bronchioles.

The apparent discontinuity between surface concentration in generations 15 and 16 is due primarily to the vast difference in clearance half times between the two generations, to the assumption that mucociliary transport suddenly stops at generation 15 , and to the symmetric nature of the Weibel model which sets the respiratory bronchioles at the same distance from the trachea regardless of their location within the lungs. In reality there is probably still a considerable increase in retention in the regions of the respiratory bronchioles, though the increase may be more gradual between the ciliated and non-ciliated airways.

The effects on long-term retention of variations in alveolar clearance are straightforward. Longer retention half times result in longer times to achieve equilibrium, higher equilibrium retention, and higher concentrations and doses to the alveolar spaces. Particle size and breathing pattern will alter the magnitude of the equilibrium retention according to the dependence of deposition on these factors. Figures 5 and 6 show the effects of particle size and 
breathing pattern. The larger the particle size and the higher the flow rate, the more peaked the surface concentration and integrated dose distributions will be in the vicinity of the large airways.

The results shown in figs 5 and 6 represent the distribution of particle number concentration and integrated dose assuming identical particle number concentration in the inspired air. If the same number of particles are deposited from each breath regardless of their volume, however, the surface mass concentration of deposited $10 \mu \mathrm{m}$ particles will be five orders of magnitude larger than the $0.5 \mu \mathrm{m}$ case. Similarly, if the same mass is deposited in each breath for all cases, the surface number concentration of deposited particles for the $10 \mu \mathrm{m}$ case will be three orders of magnitude smaller than the $0.5 \mu \mathrm{m}$ case. Since the effects of an inhaled aerosol may depend on either surface number concentration or surface mass concentration, these differences should be kept in mind.

These calculations suggest that long-term inhalation of toxic particles will produce its major effect on respiratory bronchioles. Should the mucus transport mechanism be impaired, however, the site of highest risk could easily shift from the respiratory bronchioles to the large airways. Calculations show that a tenfold decrease in transport rate would increase the surface concentration in the large airways to levels equal to that in the respiratory bronchioles.

The pattern of surface concentration at equilibrium can be contrasted with that after an acute exposure in which Gerrity et $a^{l^{1}}$ calculated that the highest surface concentration occurs at generation 3 . Equation $21 \mathrm{~A}$, though, predicts that the timeintegrated dose of an acute exposure numerically equals the surface concentration at equilibrium of a continuous exposure divided by the breathing frequency $\mathrm{f}$. Since the reactivity time of inhaled toxins is important in assessing the health effects of inhaled particles, the actual patterns of risk in the living lung may lie between the acute and continuous surface concentration patterns of the model.

The total lung burden at equilibrium is predicted to be $9 \%$ of the total number of particles deposited continuously in one year. An intermittent exposure (simulating an occupational exposure) over a 25-year period is predicted to result in a whole lung retention that is $1.5 \%$ of the total number of particles entering the lung during that time. The ashed lungs of workers exposed to silica have been found to retain $2 \%-5 \%$ of their total occupational exposure. ${ }^{30}$

In summary, the present model of retention is capable of predicting both whole lung and regional accumulation and retention of continuously inhaled particles. Even though only one particle species was considered, the model is applicable to a wide range of particle types and sizes. The calculated whole lung equilibrium burdens are consistent with the sparse experimental information available. The kinetics of retention in the model predict that the large airways and respiratory bronchioles are most likely to evince physiological or pathological responses to continuously inhaled particles. This may also account, in part, for the focal nature of many pulmonary diseases.

Although this model uses many assumptions and complex calculations, it is based on the best available data on particle deposition and clearance and thus represents a rational approach to the problems of lung dosimetry of inhaled particles.

\section{Appendix}

We first consider pathway " $a$ ". The retention of particles in each ciliated airway generation depends on the rate at which particles enter the generation from distal generations and from the external environment. The retention also depends on the rate at which particles are transported from the generation on the mucus blanket. The net rate of change of particles in generation $\alpha$ due to deposition in generation $\beta$ is given by the following differential equation:

$$
\frac{d N_{\alpha \beta}^{a}(t)}{d t}= \begin{cases}-\lambda_{\alpha} N_{\alpha \beta}^{a}(t) & (\alpha<\beta \leqslant 15) \\ +\lambda_{\alpha+1} N_{\alpha+1, \beta}^{a}(t) & (x=\beta \leqslant 15) \\ -\lambda_{\alpha} N_{\alpha \alpha}^{a}(t)+f R_{\alpha} & (\text { all other } \alpha, \beta) \\ 0 & \end{cases}
$$

where $\lambda_{\alpha}=v_{\alpha} l_{\alpha}, l_{\alpha}$ is the length of an airway in generation $\alpha, f$ is the respiration rate in breaths a minute, and $R_{\beta}$ is the fraction of particles deposited in generation $\beta$ during one respiratory cycle. This equation is an adaptation to continuous deposition and clearance of an equation derived by Lee et $\mathbf{l}^{17}$ for mucociliary clearance of acutely deposited particles. The part of eq 1A that governs the rate of change of particle number for the case of $\alpha<\beta$ states that the net rate of change is the difference between the rate at which particles enter from distal generations by mucus transport and the rate at which particles leave by mucus transport. For the case of $\alpha=\beta$, the rate is governed by the rate particles leave by mucus transport and the rate particles enter by deposition. The case $\alpha>\beta$ is merely a statement that all particles are transported cephalad. Fundamental to eq $1 \mathrm{~A}$ is the idea that the rates of particle entry and exit are proportional to particle number. It should also be emphasised that eq $1 \mathrm{~A}$ and subsequent equations are approximations. The initial conditions for eq $1 \mathrm{~A}$ are

$$
\mathrm{N}_{\alpha \beta}^{\mathrm{a}}(0)=0 \quad(\text { all } \alpha, \beta)
$$


With these initial conditions, eq $1 \mathrm{~A}$ has the solution

$$
\mathrm{N}_{\alpha \beta}^{\mathrm{a}}(\mathrm{t})=\frac{\mathrm{f}}{\lambda_{\alpha}} \begin{cases}\mathrm{R}_{\beta}\left\{\sum_{j=\alpha+1}^{\beta}\left(\mathrm{e}^{-\lambda_{\alpha} t}-\mathrm{e}^{-\lambda_{\mathrm{j}}}\right) \prod_{\substack{k=\alpha \\(\mathbf{k} \neq j)}}^{\beta} \lambda_{\mathbf{k}}\left(\lambda_{\mathbf{k}}-\lambda_{\mathrm{j}}\right)^{-1}+\left(1-\mathrm{e}^{-\lambda_{\alpha}}\right)\right\} & (\alpha<\beta \leqslant 15) \\ \mathrm{R}_{\alpha}\left(1-\mathrm{e}^{-\lambda_{\alpha} t}\right) & (\alpha=\beta) \\ 0 & (\text { all other } \alpha, \beta)\end{cases}
$$

For pathway " $b$ " the net rate of change of particles in generation $\alpha$ due to deposition in generation $\beta$ is given by

$$
\frac{\mathrm{dN}_{\alpha \beta}^{\mathrm{b}}(\mathrm{t})}{\mathrm{dt}}=\left\{\begin{array}{cl}
-\lambda_{\alpha} \mathrm{N}_{\alpha \beta}^{\mathrm{b}}(\mathrm{t}) & (\alpha \leqslant 15, \beta>15) \\
+\lambda_{\alpha+1} \mathrm{~N}_{\alpha+1, \beta}^{\mathrm{b}}(\mathrm{t}) & \\
-\lambda_{\alpha} \mathrm{N}_{\alpha \alpha}^{\mathrm{b}}(\mathrm{t})+0 \cdot 4 \mathrm{fR}_{\alpha} & (\alpha=\beta>15) \\
0 & (\text { all other } \alpha, \beta)
\end{array}\right.
$$

This equation has the same form as eq $1 \mathrm{~A}$ except that when $\alpha>15, \lambda_{\alpha}$ is the clearance constant of the rapid component of alveolar clearance equal to $4.8 \times 10^{-4}$ $\mathrm{min}^{-1}$. The deposition term in eq $4 \mathrm{~A}$ is multiplied by 0.4 since this is the fraction of deposited particles cleared by the rapid component. With the same initial conditions given by eq $2 \mathrm{~A}$, the solution of eq $4 \mathrm{~A}$ is

$$
\mathbf{N}_{\alpha \beta}^{\mathrm{b}}(\mathrm{t})=\frac{0.4 \mathrm{f}}{\lambda_{\alpha}} \begin{cases}\mathrm{R}_{\beta}\left\{\sum_{\mathrm{j}=\alpha+1}^{16}\left(\mathrm{e}^{-\lambda_{\alpha} t}-\mathrm{e}^{-\lambda_{j} t}\right) \prod_{\substack{k=\alpha \\ \mathbf{k} \neq j)}}^{16} \lambda_{\mathbf{k}}\left(\lambda_{\mathbf{k}}-\lambda_{\mathrm{j}}\right)^{-1}+\left(1-\mathrm{e}^{-\lambda_{\alpha} t}\right)\right\} & (\alpha \leqslant 15, \beta>15) \\ \mathrm{R}_{\alpha}\left(1-\mathrm{e}^{-\lambda_{\alpha} \mathrm{t}}\right) & (\alpha=\beta>15)(5 \mathrm{~A}) \\ 0 & \text { (all other } \alpha, \beta)\end{cases}
$$

Equation $5 \mathrm{~A}$ is similar to eq $3 \mathrm{~A}$ except that the summation and product extend only to 16 rather than to $\beta$. This results from assuming that particles in any alveolar generation move directly to the terminal bronchioles without passing through any intervening alveolated generations.

Finally, the governing equation for the rate of change of particles deposited in the alveolated generations and cleared by the slow component of alveolar clearance is

$$
\frac{\mathrm{dN}_{\alpha \beta}^{\mathrm{c}}(\mathrm{t})}{\mathrm{dt}}= \begin{cases}-\Lambda_{\alpha} \mathrm{N}_{\alpha \alpha}^{\mathrm{c}}(\mathrm{t})+0.6 \mathrm{fR}_{\alpha} & (\alpha=\beta>15) \\ 0 & \text { (all other } \alpha, \beta)\end{cases}
$$

where $\Lambda_{\alpha}$ is the clearance constant of the slow component and equals $7 \cdot 1 \times 10^{-6} \mathrm{~min}^{-1}$. With the same initial conditions given by eq $2 \mathrm{~A}$, the solution to eq $6 \mathrm{~A}$ is

$$
\mathrm{N}_{\alpha \beta}^{\mathrm{c}}(\mathrm{t})= \begin{cases}\frac{0.6 \mathrm{fR}}{\Lambda_{\alpha}}\left(1-\mathrm{e}^{-\Lambda_{\alpha} t}\right) & (\alpha=\beta>15) \\ 0 & (\text { all other } \alpha, \beta)\end{cases}
$$

The retention of particles as a function of time in any generation $\alpha$ due to all pathways is obtained by summing over all $\beta \geqslant \alpha$ :

$$
N_{\alpha}(t)=\sum_{\alpha=\beta}^{23}\left\{N_{a \beta}^{a}(t)+N_{a \beta}^{b}(t)+N_{a \beta}^{c}(t)\right\}
$$

Total lung retention as a function of time is then obtained by summing over $\alpha$ :

$$
N^{\text {tot }}(t)=\sum_{\alpha=0}^{23} N_{\alpha}(t)
$$

With these solutions for the retention of continuously inhaled particles, we can calculate other quantities which give a measure of the exposure of the lung regionally to particles. The simplest quantity to calculate is the surface concentration of particles as a function of time. This is given by

$$
C_{\alpha}(t)=N_{\alpha}(t) / A_{\alpha}
$$

where $A_{\alpha}$ is the surface area of generation $\alpha$ calculated using the dimensions of the Weibel model.

The number of particles per unit surface area that pass over the tissue in generation $\alpha$ in a time $t$ is given by the following integrated dose:

$$
D_{\alpha}(t)=\int_{0}^{t} C_{\alpha}(t) d t
$$


For pathway " $a$ " the integrated dose is:

$$
\begin{aligned}
D_{\alpha}^{a}(t)= & \frac{f}{\lambda_{\alpha} A_{\alpha}}\left\{R_{\alpha}\left(t+\frac{e^{-\lambda_{\alpha} t}-1}{\lambda_{\alpha}}\right)+\sum_{\beta=\alpha+1}^{15} R_{\beta}\left\{\sum_{j=\alpha+1}^{\beta}\left(\frac{e^{-\lambda_{j} t}-1}{\lambda_{j}}-\frac{e^{-\lambda_{\alpha} t}-1}{\lambda_{\alpha}}\right)\right.\right. \\
& \left.\left.X \prod_{\substack{k=\alpha \\
(k \neq j)}}^{\beta}\left(\lambda_{k}-\lambda_{j}\right)^{-1} \lambda_{k}+t+\frac{e^{-\lambda_{\alpha} t}-1}{\lambda_{\alpha}}\right\}\right\} \quad(\alpha \leqslant 15)
\end{aligned}
$$

For pathway " $b$ " the integrated dose is

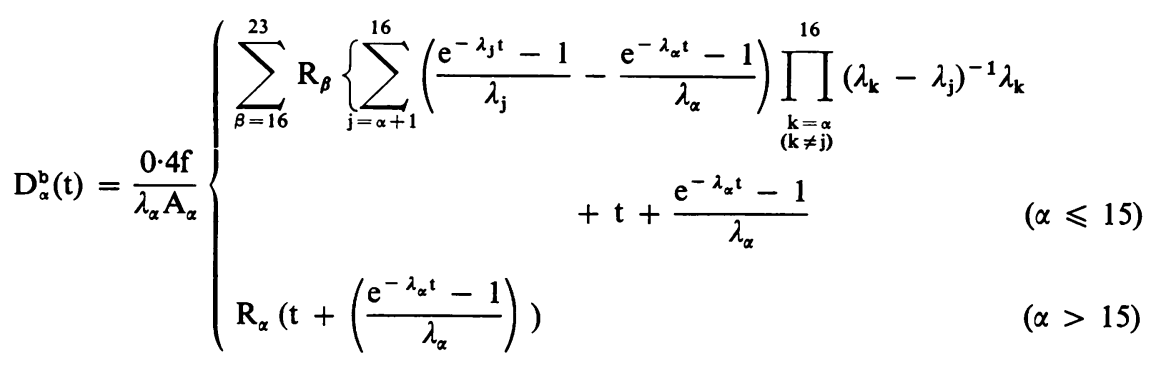

For pathway " $c$ " the integrated dose is

$$
\mathrm{D}_{\alpha}^{\mathrm{c}}(\mathrm{t})=\frac{0.6 \mathrm{f} \mathrm{R}_{\alpha}}{\Lambda_{\alpha} \mathrm{A}_{\alpha}}\left(\mathrm{t}+\frac{\mathrm{e}^{-\Lambda_{\alpha} \mathrm{t}}-1}{\Lambda_{\alpha}}\right)
$$

One of the results of this model is the feature that during a continuous exposure the rate of particle clearance from the lung approaches the rate of particle deposition. When this occurs, the total number of retained particles in each generation reaches a constant which is calculated by allowing $t \rightarrow \infty$ in eqs $3 \mathrm{~A}, 5 \mathrm{~A}$, and $7 \mathrm{~A}$. The resulting equilibrium retentions are

$\mathrm{N}_{\alpha}^{\mathrm{a}}(\mathrm{t} \rightarrow \infty) \simeq \frac{\mathrm{f}}{\lambda_{\alpha}} \sum_{\beta=\alpha}^{15} \mathrm{R}_{\beta} \quad(\alpha \leqslant 15)$

for pathway "a",

$\mathrm{N}_{\alpha}^{\mathrm{b}}(\mathrm{t} \rightarrow \infty) \simeq \frac{0.4 \mathrm{f}}{\lambda_{\alpha}} \begin{cases}\sum_{\beta=16}^{23} \mathrm{R}_{\beta} & (\alpha \leqslant 15) \\ \mathrm{R}_{\alpha} & (\alpha>15)\end{cases}$

for pathway " $b$ ", and

$\mathrm{N}_{\alpha}^{\mathrm{c}}(\mathrm{t} \rightarrow \infty) \simeq \frac{0.6 \mathrm{fR}_{\alpha}}{\Lambda_{x}} \quad(\alpha>15)$

for pathway "c".

Similarly, the particle surface concentrations reach an equilibrium. The integrated doses, on the other hand, increase linearly with time as $t \rightarrow \infty$. These asymptotic expressions are

$\mathrm{D}_{\alpha}^{\mathrm{a}}(\mathrm{t} \rightarrow \infty) \simeq \frac{\mathrm{ft}}{\lambda_{\alpha} \mathrm{A}_{\alpha}} \sum_{\beta=\alpha}^{15} \mathrm{R}_{\alpha} \quad(\alpha \leqslant 15)$

for pathway " a",

$D_{a}^{b}(t \rightarrow \infty) \simeq \frac{0 \cdot 4 \mathrm{ft}}{\lambda_{\alpha} \mathrm{A}_{\alpha}} \begin{cases}\sum_{\beta=16}^{23} \mathrm{R}_{\alpha} & (\alpha \leqslant 15) \\ \mathrm{R}_{\alpha} & (\alpha>15)\end{cases}$

for pathway "b", and

$D_{a}^{c}(\mathrm{t} \rightarrow \infty) \simeq \frac{0.6 \mathrm{fR}_{\alpha} \mathrm{t}}{\Lambda_{\alpha}} \quad(\alpha>15)$

for pathway "c".

It is also interesting to note the integrated dose over all time from an acute deposition equals the equilibrium concentration divided by the breathing frequency-that is,

$D_{\alpha}^{\text {acute }}(t \rightarrow \infty)=\int_{0}^{\infty} C_{\alpha}^{\text {acute }}(t) d t=C_{\alpha}(t \rightarrow \infty) / f$

where $C_{\alpha}^{\text {acute }}(\mathrm{t})$ is given by Lee et al. ${ }^{17}$ 


\section{References}

${ }^{1}$ Cohen D. Ferromagnetic contamination in the lungs and other organs of the human body. Science 1973;180:745-8.

${ }^{2}$ Cohen D, Kaufman LA, Givler E, Crowther T. Various phenomena of the lung's magnetic field. In report of the low field group: The magnetic field of the lung. Springfield, Virginia: National Technical Information Service, 1978. (MIT/FBNML78/1 1978.)

${ }^{3}$ Kalliomäki P, Karp PJ, Katla T, Mäkipää P, Sarr P, Tossavainen A. Magnetic measurements of pulmonary contamination. Scand J Work Environ Health 1976;4:232-9.

${ }^{4}$ Altschuler B, Yarmus L, Palmes ED, Nelson N. Aerosol deposition in the human respiratory tract. AMA Archives of Industrial Health 1957;15:293-303.

${ }^{5}$ Altschuler B, Palmes ED, Yarmus L, Nelson N. Intrapulmonary mixing of gases studied with aerosol. J Appl Physiol 1959;14:321-7.

${ }^{6}$ Altschuler B. Calculation of regional deposition of aerosol in the respiratory tract. Bulletin of Mathematical Biophysics 1959;21:275-80.

${ }^{7}$ Beeckmans JM. The deposition of aerosols in the respiratory tract. I. Mathematical analysis and comparison with experimental data. Canadian Journal of Pharmacology 1965;43:157-72.

${ }^{8}$ Beeckmans JM. Correction factor for size selective sampling results, based on a new computed alveolar deposition curve. Ann Occup Hyg 1965;8:221-31.

${ }^{9}$ Findeisen W. Uber das absetzen kleiner, in der luft suspendierte teilchen in der menschlichen lunge bei der atmung. Pfluger Archiv für die Gesamte Physiologie des Menschen und der Tiere 1935;236:367-79.

${ }^{10}$ Landahl HD. On the removal of airborne droplets by the human respiratory tract: I. The lung. Bulletin of Mathematical Biophysics 1950;12:43-6.

${ }^{11}$ Taulbee DB, Yu CP. A theory of aerosol deposition in the human respiratory tract. J Appl Physiol 1975;38:77-85.

${ }_{12}$ Taulbee DB, Yu CP, Heyder J. Aerosol transport in the human lung from analysis of single breaths. J Appl Physiol $1978 ; 44: 803-12$

${ }^{13} \mathrm{Yu}$ CP, Taulbee DB. A theory of predicting respiratory tract deposition of inhaled particles in man. In: Walton WH, ed. Inhaled particles IV, Part 1. New York: Pergamon Press, 1977:35-7.

${ }^{14}$ Brain JD, Valberg PA. Model of lung retention based on ICRP task group report. Arch Environ Health 1974;28:1-11.

${ }^{15}$ Task group on lung dynamics. Deposition and retention models for internal dosimetry of the human respiratory tract. Health Physics 1966;12:173-207.

${ }^{16}$ Weibel ER. Morphometry of the human lung. New York: Academic Press, 1963.

${ }^{17}$ Lee PS, Gerrity TR, Hass FJ, Lourenco RV. A model for tracheobronchial clearance of inhaled particles in man and a comparison with data. IEEE Trans Biomed Eng 1979; BME26(11):624-30.

${ }^{18}$ Morrow PE. Alveolar clearance of aerosols. Arch Intern Med 1973;131:101-8.

${ }^{19}$ Mercer TT. On the role of particle size in the dissolution of lung burdens. Health Physics 1967;13:1211-21.

${ }^{20}$ Morrow PE, Gibb FR, Johnson L. Clearance of insoluble dust from the lower respiratory tract. Health Physics 1964;10: 543-55.

${ }^{21}$ Gerrity TR, Lee PS, Hass FJ, Marinelli A, Werner P, Lourenco RV. Calculated deposition of inhaled particles in the airway generations of normal subjects. J Appl Physiol 1979;47:867-73.

22 Yeates DB, Aspin N, Levison H, Jones MT, Bryan AC. Mucociliary tracheal transport in man. J Appl Physiol 1975;39:487-95.

${ }^{23}$ Yeates DB, Gerrity TR, Garrard CS. Characteristics of tracheobronchial deposition and clearance in man. In: Walton WH, ed. Inhaled particles V. Oxford: Pergamon Press, 1982.

${ }^{24}$ Wilkey DD, Lee PS, Hass FJ, Gerrity TR, Yeates DB, Lourenco RV. Mucociliary clearance of deposited particles from the human lung: Intra- and intersubject reproducibility, total and regional lung clearance, and model comparison. Arch Environ Health 1980;35:294-302.

${ }^{25}$ Lippmann M, Yeates DB, Albert RE. Deposition, retention and clearance of inhaled particles. Br J Ind Med 1980;37:337-62.

${ }^{26}$ Asmundsson TH, Kilburn KH. Mucociliary clearance rate at various levels in dog lungs. Am Rev Respir Dis 1970; 102:388-97.

${ }^{27}$ Albert E, Lippmann M, Spiegelman J, et al. The clearance of radioactive particles from the human lung. In: Davies CN, ed. Inhaled particles III. New York: Pergamon Press, 1967:361-77.

${ }^{28}$ Gibb FR, Morrow PE. Alveolar clearance in dogs following inhalation of an iron-59 oxide aerosol. J Appl Physiol 1962;71:429-32.

${ }^{29}$ Stahlhoffen W, Gebhart J, Heyder J. Experimental determination of the regional deposition of aerosol particles in the human respiratory tract. Am Ind Hyg Assoc J 1980;41:385-98a.

${ }^{30} \mathrm{McCrae} \mathrm{J}$. The ash of silicotic lung. South African Institute of Medical Research, 1913;117-22. 